\title{
Experimental and Numerical Studies of Jaw Crusher Supporting Structure Fatigue Failure
}

\author{
Eugeniusz Rusiński - Przemysław Moczko* - Damian Pietrusiak - Grzegorz Przybyłek \\ Wroclaw University of Technology, Poland
}

\begin{abstract}
In this paper, studies into the causes of fatigue cracks of a jaw crusher supporting structure are presented. The problems appeared after short time of operation at a new crushing facility. A large number of fatigue cracks with a high growth rate and bolt failures were observed in the crusher supporting structure. Considering the high dynamic forces that occur during the operation of such equipment, an investigation into the problem was undertaken in order to prevent catastrophic failure of the crushing station. A specially developed, combined numerical and experimental method was used to determine the reasons for the problems and to solve them.
\end{abstract}

Keywords: jaw crushers, fatigue resistance, resonance, numerical simulations, experimental methods

\section{INTRODUCTION}

High dynamic forces are present during the operation of crushing stations. The entire load is transferred to the supporting structures and foundations during operation. Such loads have to be considered at the design stage and special care must be paid to modal parameters of the structure in order to prevent possible resonance problems. The present state of the art concerning static load is well developed and provides full resistance in terms of the ultimate strength range of the structures [1] and [2]. Design with a fatigue resistance approach is still problematic due to the complexity of the fatigue phenomenon. Additionally, vibration and, in some cases, resonance problems intensify the occurrence of the fatigue fractures. Although this problem has been known for many years, its complete treatment has become possible by the common use of sophisticated experimental and numerical techniques [3] and [4]. There are a lot of examples of failures caused by unpredictable dynamic loads [5] and [6]. Reliable assessment and a proper solution are now possible due to the complex numerical-experimental approach, which is presented in Fig. 1. This procedure can be used for various cases of failures. The modified procedure can be adapted for use in the design stage of new structures as well.

Application to a real structure failure was carried out at one of the Polish mines. The crushing station consists of typical equipment such as a chute, conveyor system, hammer and jaw crusher, which is supported by a steel frame anchored to the concrete foundations. A view of the crushing station is shown in Fig. 2.

Within a short time (few weeks) of starting operations at the new facility, a number of fatigue cracks and bolt damage to the crusher supporting structure were observed. A view of the cracks in the side vertical bracing is shown as an example in Fig. 3.

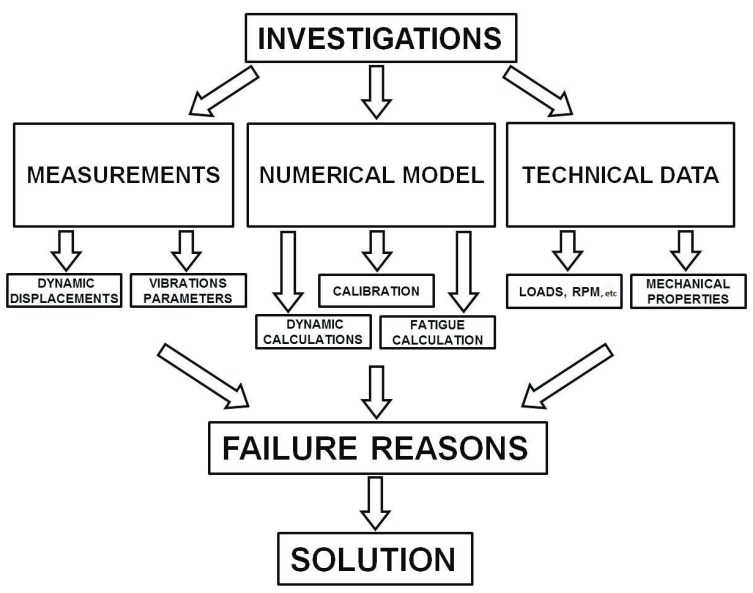

Fig. 1. Investigation procedure for determining the reasons for failure

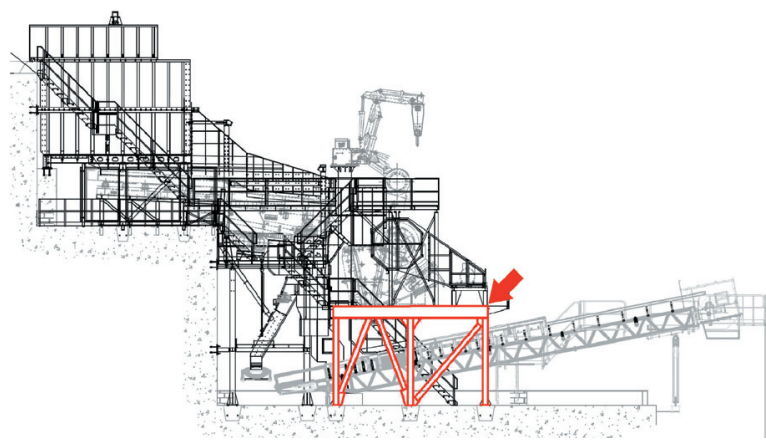

Fig. 2. Jaw crusher layout (supporting structure highlighted in red, marked with arrow)

The growth rate of fatigue cracks was high. The maximum length of cracks was found to be $160 \mathrm{~mm}$. The significant level of damage meant that immediate action would have to be taken in order to prevent 
irreversible damage to the whole structure. But first the cause of the damage had to be identified.

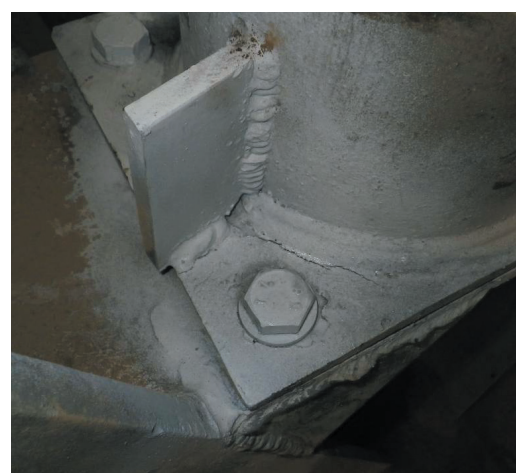

Fig. 3. Fatigue crack in the side bracing

A combined numerical and experimental method was used to determine the reasons for the poor durability of the structure and to resolve them. In the next sections, the various investigations are presented.

\section{EXPERIMENTAL AND NUMERICAL STUDIES}

The first part of study covers measurements of dynamic parameters of the structure and an estimation of the dynamic loads causing the fractures. Dynamic loads acting on the crusher frame have a stochastic character, while the material provided to the crusher varies in size and mechanical properties in a random way. This situation required a determination of the maximum possible load acting on the structure in experimental terms. The measured loads were then used for the numerical strength calculations of the crusher's supporting structure. In the following subsections, the results of both approaches are presented.

\subsection{Experimental Tests}

The first part of investigations was aimed at measuring the dynamic behaviour of the structure and estimating the dynamic loads causing the fractures. Measurements of the displacement vibrations of the supporting frame were carried out using the following equipment:

- $\quad$ vibrometer - COMMTEST vb7,

- $\quad$ scanning vibrometer - POLYTEC PSV-400.

Tests were performed at two points (A, B, Fig. 4), where displacements of the structure were measured in two horizontal directions, $x$ longitudinal and $y$ transverse.

The testing equipment used during the tests is shown in Figs. 5 and 6.

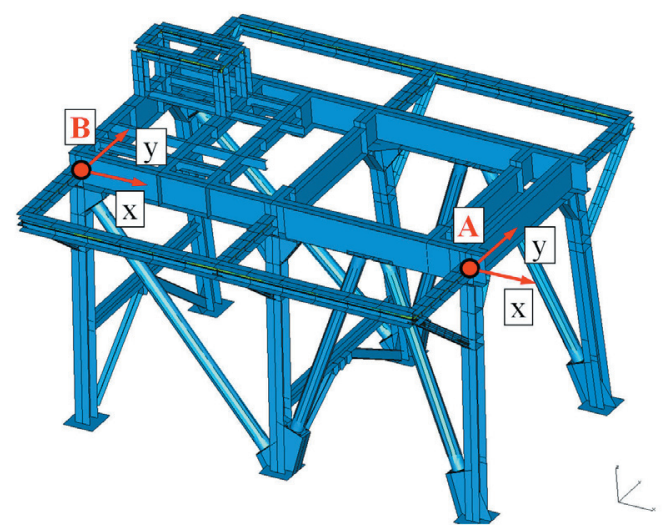

Fig. 4. Locations and directions of the measuring points

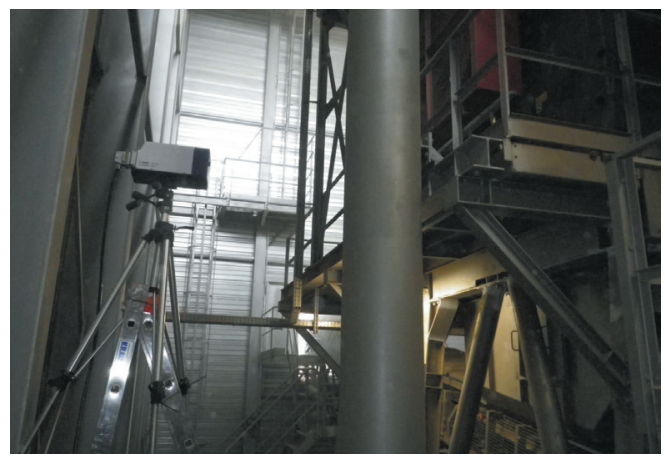

Fig. 5. Scanning head of the POLYTEC PSV-400 vibrometer

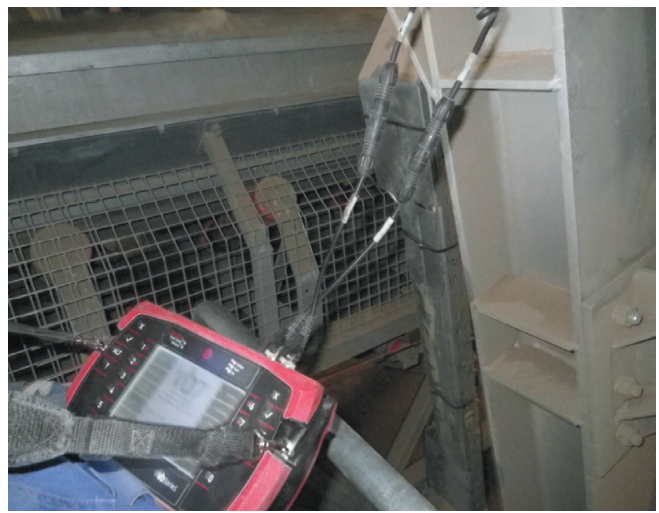

Fig. 6. COMMTEST vb7 vibrometer

As a result of the measurements, a vibration spectrum and time traces were obtained. An example of a graph of the measurements results is shown in Fig. 7. The transverse vibrations of the structure are caused by resonance effects, which can be seen in the time traces of the vibrations where a characteristic beat effect is present.

Analysis of the measurement results led to the conclusion that the structure operates at a resonance condition close to the excitation frequency $f_{e x}=3.33$ $\mathrm{Hz}$. 

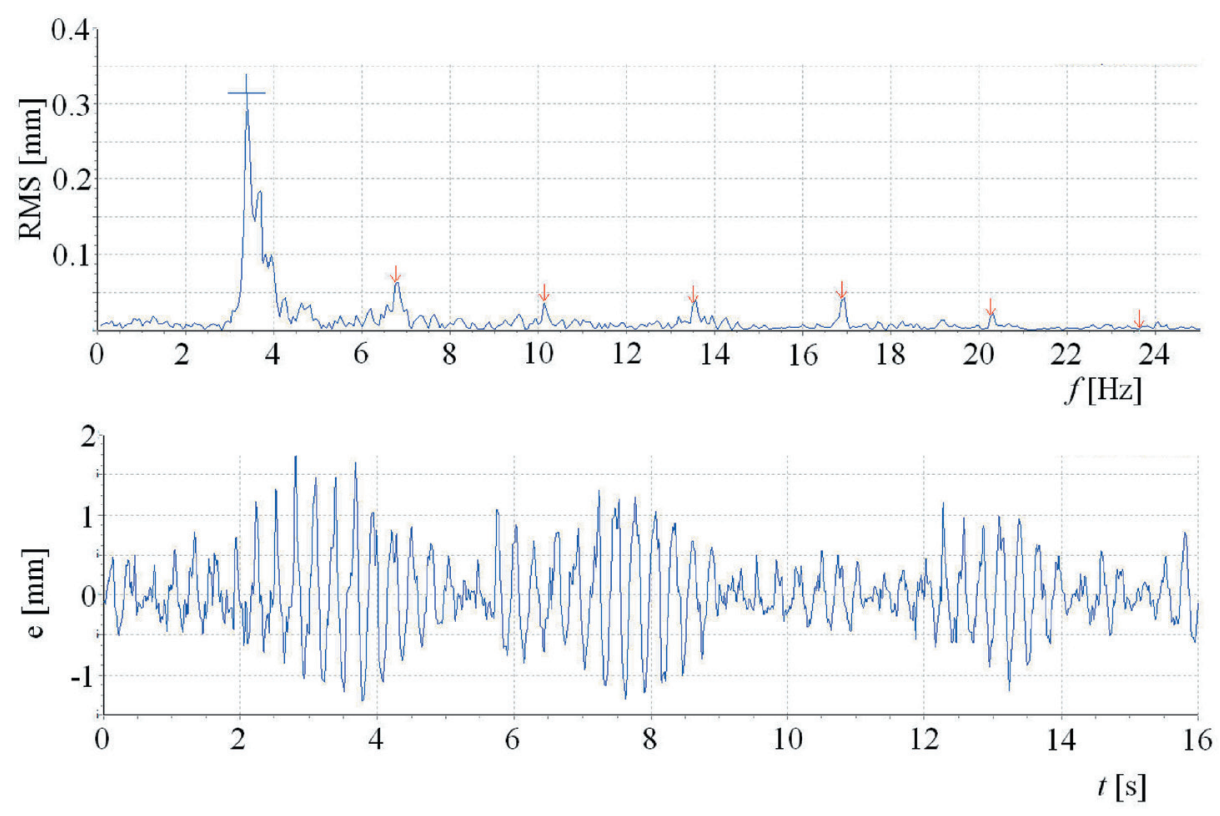

Fig. 7. Time and spectrum trace with a visible resonance effect - transverse displacement

The maximum amplitudes of the displacements measured during the tests are presented in the Table 1.

Table 1. Maximum amplitudes of the displacements

\begin{tabular}{ccc}
\hline \multirow{2}{*}{ Description } & \multicolumn{2}{c}{ Displacement [mm] } \\
\cline { 2 - 3 } & $x$ (longitudinal) & $y$ (transverse) \\
\hline Displacement measurements & 1.12 & 1.92 \\
\hline
\end{tabular}

The displacements were used to calibrate the FEM model, which was created to evaluate the dynamic behaviour and strength of the structure in its existing and modified form.

\subsection{FEM Calculations}

In order to estimate the influence of the measured vibrations and to calculate the dynamic loads causing such effects, the FEM [7] model of the supporting structure was created. The model was used as a calibration tool for the dynamic forces caused by crushing cycles. Based on the results of the calibration calculations such forces were estimated.

As a first step, a numerical model of the crusher's supporting structure was created. Shell modelling was used to evaluate the exact stress effort and deformation of the structure. Such an approach enables the calculation of stress level in the notches, which is impossible with the use of beam modelling. Fig. 8 presents a model of the supporting structure with the shell elements generated. The model consists of a linear shell, beam, as well as rigid and spring elements. Mass elements (squares) simulate the selfweight of the equipment placed on the supporting structure, such as the crusher unit, electrical motor, etc. There are 58000 elements and a similar number of nodes generated in total in the model of the structure. The boundary conditions consist of restraints and loads. Supporting points of the entire structure were restrained to simulate the behaviour of the real structure. The loads applied in the simulations are based on the results of the measurements and are described in the section below.

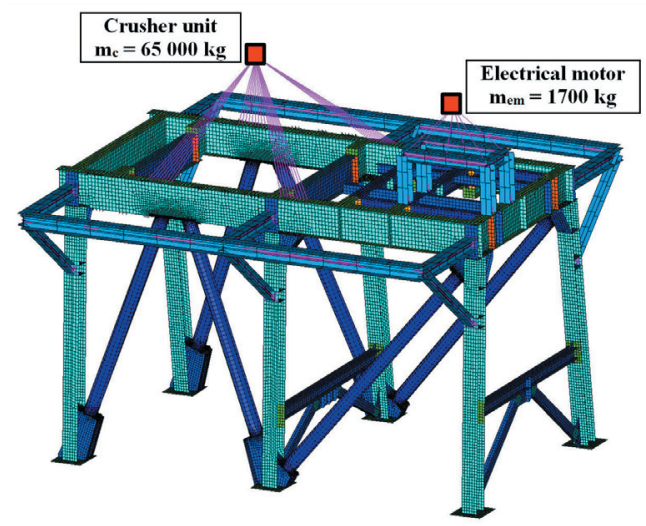

Fig. 8. Numerical FEM model of the supporting structure

Numerical simulations were aimed at confirming the dynamic behaviour of the structure using modal 
analysis and calculating and applying dynamic loads to perform strength calculations.

Numerical modal analysis of the model confirmed the presence of the resonance effect under operating conditions. Table 2 lists the frequencies of the first three modes. Fig. 9 presents the deformation plot of the first mode, which is responsible for the resonance vibrations. The direction of the vibrations is in line with those measured on site and the frequency is close to the excitation load $\left(f_{e x}\right)$, which is $f_{e x}=3.33 \mathrm{~Hz}$, $f_{1}=3.39 \mathrm{~Hz}, f_{e x}<\sim f_{1}$.

Table 2. Natural frequencies of the crusher's supporting structure

\begin{tabular}{cc}
\hline No. of natural mode & Frequency $[\mathrm{Hz}]$ \\
\hline 1 & 3.39 \\
\hline 2 & 5.96 \\
\hline 3 & 14.50 \\
\hline
\end{tabular}

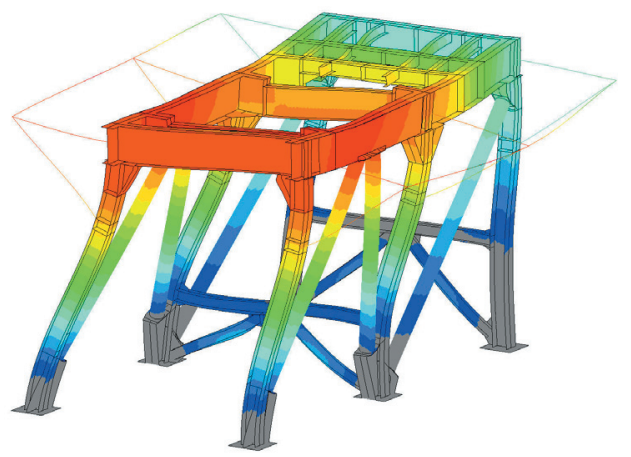

Fig. 9. Natural frequency mode shape of the crusher's supporting structure - Mode 1

The next step was to perform fatigue calculations. Considering that dynamic forces are created in the jaw area of the crusher unit, loads in both horizontal directions ( $x$ and $y$ ) were applied. Based on the displacement results, multiplication factors were calculated to obtain the same values for the displacements as those measured on site. For such conditions, strength (fatigue) calculations were performed in consideration of ISO 5049-1 standard requirements [8]. The structure of the crusher support was considered to be class $\mathrm{C}$ (number of fatigue cycles exceeds $6 \times 105)$. The steel grade used for the structure was considered to be St3S (Fe 360).

The results of fatigue calculations confirmed that the areas where cracks are present on the structure are overstressed and do not have proper fatigue resistance. There was $100 \%$ correlation between the cracks locations on the real structure and those in the numerical model (an example is shown in Fig. 10).
Areas, marked with arrows on the numerical model, indicate fatigue problems.

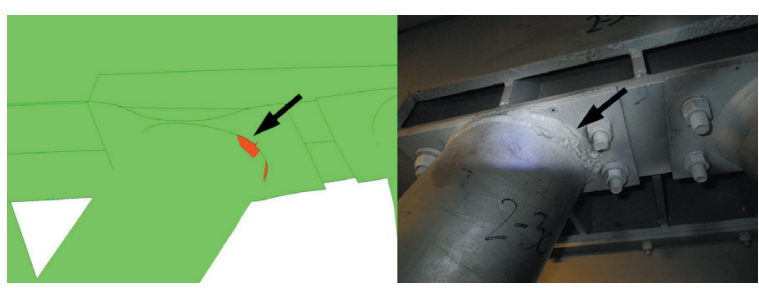

Fig. 10. Comparison of fatigue crack location in the numerical model and the existing structure

The locations of the cracks present on the crusher's supporting structure and confirmed in the numerical calculations are presented in Fig. 11.

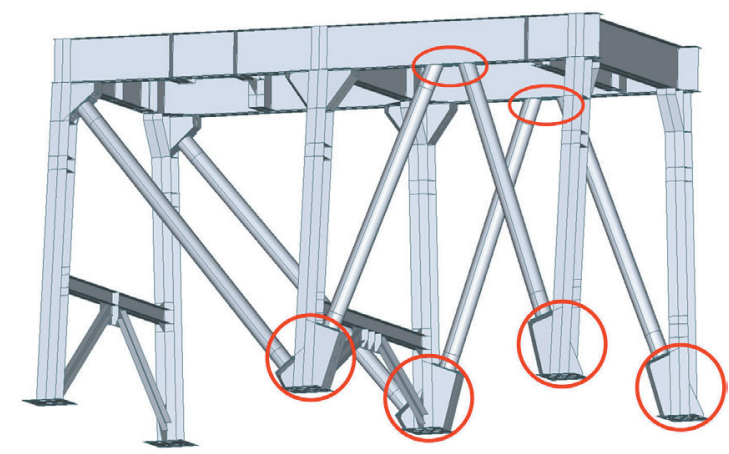

Fig. 11. Fatigue crack locations

\section{MODIFICATIONS OF THE CRUSHER SUPPORTING STRUCTURE}

Results of the test measurements as well as modal analysis and strength calculations confirmed that there are two main reasons for the poor operating conditions of the crusher supporting structure:

1) resonance vibrations in the transverse direction caused by not enough stiffness in the structure in this direction,

2) weak design of the joints, which have low resistance to loads due to operating conditions with movement in both horizontal directions (an example of such a design is shown in Fig. 12). The design of the joints would be sufficiently strong in the case of a static load, however the geometry of the gusset plates does not assure proper load transfer in the case of a dynamic load. Lateral forces acting on the faceplate and the bolted connection cause high effort in those elements. Moreover, the joints do not assure smooth stiffness changes. Therefore, strong bending and pulsations are present and cracks occur as a result of rapid fatigue. 


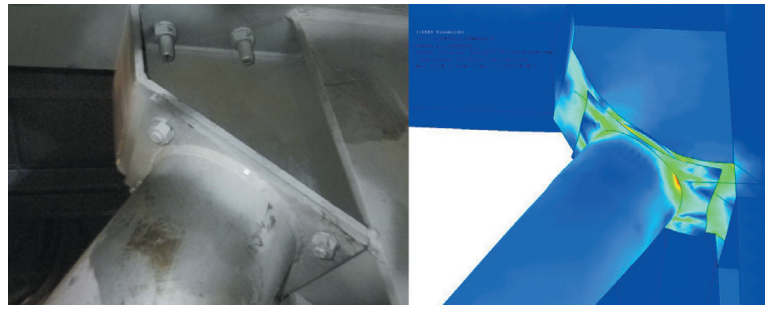

Fig. 12. Example of the weak design of the joints

The above reasons, identified by the numericalexperimental procedure, indicate the improper design of the structure, which has to be changed in order to prevent critical damage to the structure.

\subsection{Solving the Resonance Problem}

As measured on site and calculated with use of the FEM model, the structure suffers from transverse resonance vibration caused by overlapping of the excitation (crusher rpm) frequency and the first natural vibration mode. The natural frequencies must be shifted away from the excitation frequency in order to enable safe operation of the crushing station.

The basic equation for natural frequency calculations $\omega_{0}[\mathrm{~Hz}]$ is presented below:

$$
\omega_{0}=\sqrt{\frac{k}{m}}
$$

where $k$ is stiffness [N/m], and $m$ is mass [kg].

Taking into account the above equation, either the crusher support can be stiffened to increase the natural frequencies or additional mass can be placed on the frame to decrease the vibration frequency. The first solution was chosen because the cross section of the crusher supporting frame was shown not to be stiff enough in transverse direction.

The easiest way to increase stiffness would be to incorporate diagonal bracing inside the frame. However, the conveyor line located inside the frame prevents this. Therefore another stiffening solution was designed. Two of three cross-sections of the frame were reinforced with side bracing as shown in Fig. 13. Thus, there are four bracings in total anchored to the concrete foundation and welded to the existing structure. This change was incorporated into the FEM model to confirm its influence on the natural frequencies of the modified supporting structure. The position of the side bracing was mainly determined by the ease of assembly on the already existing structures. The results of the modal analysis of the modified crusher supporting structure are shown in subsection 2.3 of the paper. The results confirm that the position of the side bracing has a positive influence on the structure dynamics.

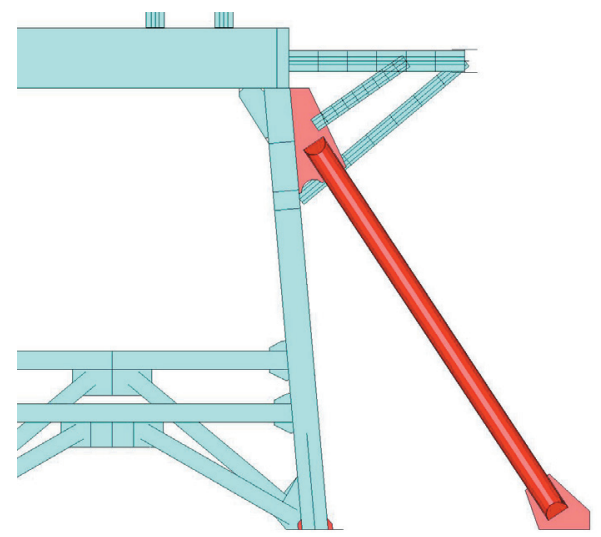

Fig. 13. Side bracing design

\subsection{Weak Joints Modifications}

According to the FEM calculations on the existing structure, the fatigue resistance of critical joints (see Fig. 11) does not fulfil ISO-5049 standards [8]. This was confirmed by the presence of the many cracks observed in these areas (an example of such cracking is shown in Fig. 3).

Due to the condition of the existing joints, which exhibit multiple cracks and thus multiple repairs, it was decided to cut out all weak joints and incorporate new redesigned ones. Special care was paid to the joint's design to assure proper fatigue resistance.

This new solution required the replacement of existing longitudinal bracings with new ones. Fig. 14 shows the new design of the longitudinal bracings incorporated into a geometrical model of the crusher supporting structure. In order to enable easy manufacturing and built process into the existing structure, welding connections were considered on the designing stage.

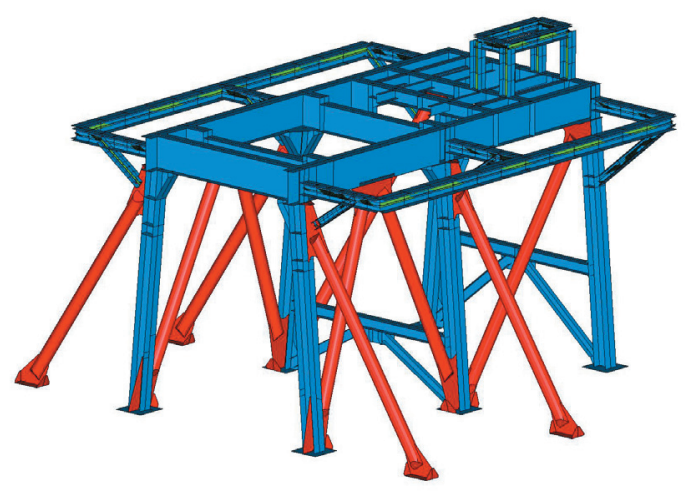

Fig. 14. New design of side and longitudinal bracings 
The supporting structure with redesigned longitudinal and side bracings was analysed with the use of FEM models to check and confirm its resistance to dynamic loads (resonance) and fatigue. The results of these analyses are presented below.

\subsection{Numerical Check Calculations of the Modified Structure}

In the first step, a modified model of the crusher supporting structure was checked for resonance issues. Modal analysis of the model confirmed that the natural frequencies of the structure were shifted significantly up. Table 3 lists the frequencies of the first three modes. Fig. 15 presents a deformation plot of the first mode, which was responsible for the resonance vibrations in the existing structure. The first natural frequency is shifted from the excitation frequency and equals: $f_{e x}=3.33 \mathrm{~Hz}, f_{1}=8.58 \mathrm{~Hz}$, $f_{\text {ex }}<f_{1}$.

There are no natural frequencies close to the first harmonic of the excitation frequency, which equals: $f_{e x, 1 h}=6.66 \mathrm{~Hz}$.

The above results confirm that there is no risk of resonance during operation of the modified supporting structure of the crusher.

Table 3. Natural frequencies of the modified crusher supporting structure

\begin{tabular}{cc}
\hline No. of natural mode & Frequency $[\mathrm{Hz}]$ \\
\hline 1 & 8.58 \\
\hline 2 & 8.72 \\
\hline 3 & 16.85 \\
\hline
\end{tabular}

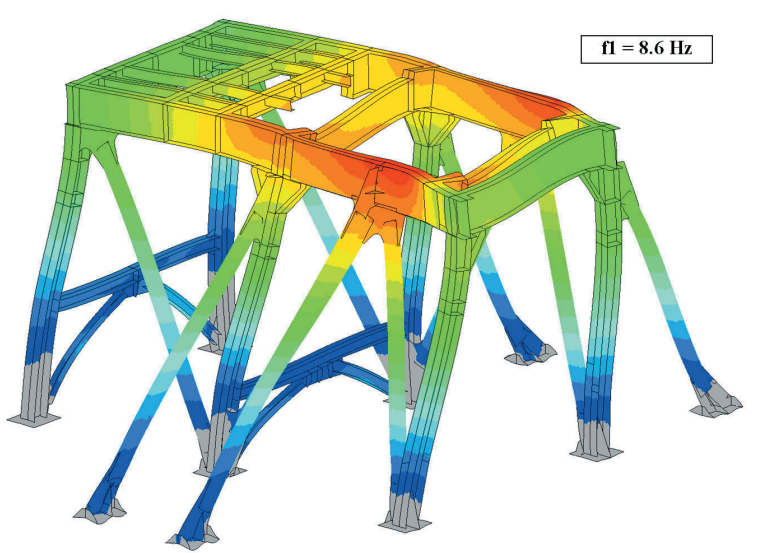

Fig. 15. Natural frequency mode of the modified crusher supporting structure - Mode 1

The next step was to perform fatigue check calculations of the modified structure to verify whether the new design of the joints shows proper fatigue resistance. Calculations were carried out using the FEM model in accordance with ISO-5049 standard requirements. As a result of such calculations, the stress amplitude $\sigma_{a}$ at each point of the structure was obtained. The maximum value calculated in the structure was found in the gusset plate of the longitudinal bracing: $\sigma_{a \max }=42 \mathrm{MPa}$.

Fig. 16 shows a view on this area with the stress amplitude plot. The maximum stress peak is located in the welded connection of the gusset plate and pipe section. In this area, a $\mathrm{K} 3$ notch was designed where the permissible stress amplitude equals: $\sigma_{\text {aperm }}=60$ $\mathrm{MPa}$

A comparison of both values confirms that the modified structure of the crusher supporting structure is safe from the fatigue resistance point of view.

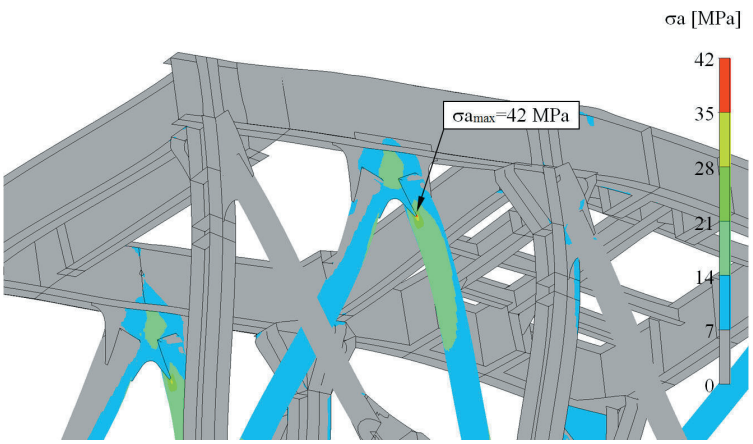

Fig. 16. Stress amplitude $\sigma_{a}$ in the modified crusher supporting structure

As a comparison of the existing and new design, Fig. 17 presents the same area of the gusset plate in the old design. The maximum stress amplitude in this area equals $\sigma_{a \max }=117 \mathrm{MPa}$.

This value exceeds the permissible values of the stress amplitude, which was indicated by the fatigue cracks in the maximum stress area.

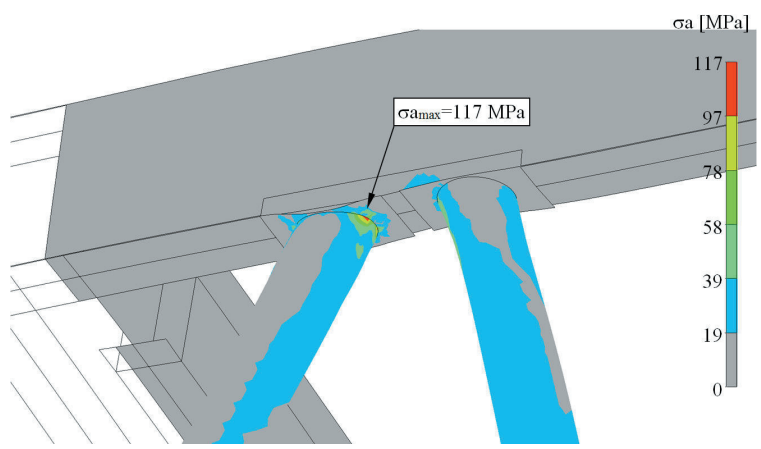

Fig. 17. Stress amplitude $\sigma_{a}$ in the existing crusher supporting structure $[\mathrm{MPa}]$ 


\section{MODIFICATIONS OF THE STRUCTURE, TESTS MEASUREMENTS}

In the previous section, the new designs of the modifications to the crusher supporting structure were presented. The results of the dynamic (modal) and fatigue calculations confirmed that the modified structure should be safe under normal operating conditions. The new design of the side and longitudinal bracings were incorporated into the structure prior to removal of existing weak joints and bracings. Fig. 18 shows an example of the redesigned joint before and after modification.

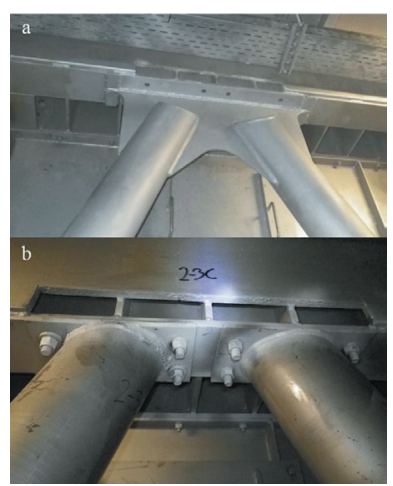

Fig. 18. Weak joint; a) after, and b) before modification

The final step of the investigation was taking measurements of the dynamic behaviour of the modified structure in order to confirm that resonance was no longer present during operation. The tests were performed with the use of a COMMTEST vb7 vibrometer at the same locations as the tests on the original structure. The vibration spectrum and time traces obtained are shown in Fig. 19. There are no resonance vibrations present in the time trace of the measured signals. The RMS value of the vibrations is $400 \%$ lower compared to the vibrations of the original structure.

\section{CONCLUSIONS}

In this paper, an experimental and numerical approach to investigating the reasons for the serious fatigue failures of the jaw crusher supporting structure is presented. The investigation method dedicated to the high performance machines was used for this purpose. Experimental vibrations measurements and FEM calculations allowed us to obtain the dynamic loads caused by the crushing process, to evaluate the influence of such loads on the fatigue resistance of the structure, and to confirm the operating conditions with the resonance effect present. Fatigue calculations confirmed that the structure shows fatigue weakness in particular joints. It must be emphasized that there is $100 \%$ correlation between the crack locations on the real structure and those in the numerical model.
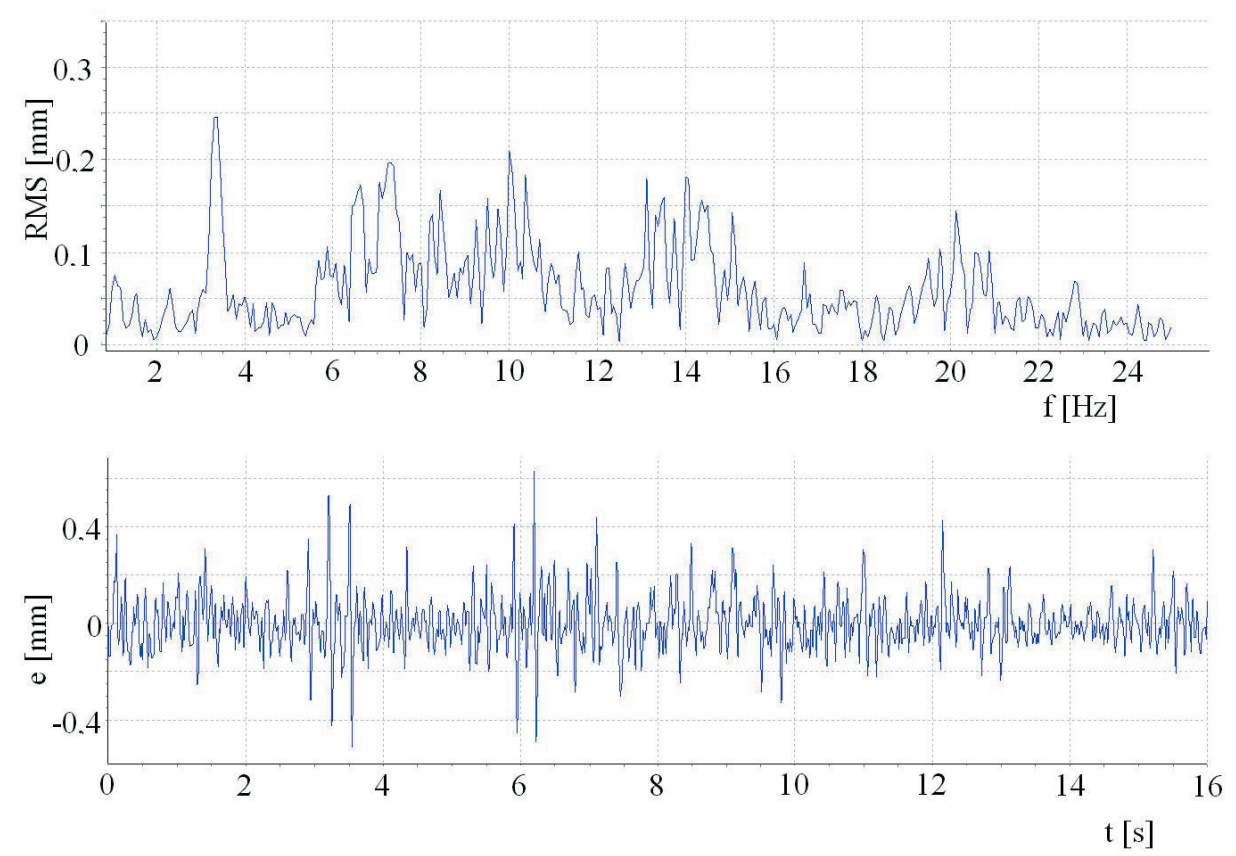

Fig. 19. Time and spectrum displacement signal of transverse vibrations of the modified structure 
In order to solve the resonance problems and to avoid fatigue cracks in the structure, additional side bracings were incorporated and existing longitudinal bracings were replaced with redesigned ones. The new design was checked for dynamic and fatigue behaviour using the modified FEM model.

In the final stage, the modified structure was tested using a vibration measuring device. The test measurements confirmed that there was no resonance and that the structure exhibited proper behaviour during normal operation.

The combined, experimental-numerical method can be applied to various operational problems of industrial equipment. This method is one way to improve design quality and can be used on new or existing structures. This method also allows us to identify reasons for failures, to measure operational parameters, to calibrate numerical models, and to investigate various phenomena causing potential problems. The verified numerical models can be used for various analyses in order to solve existing problems or to pinpoint possible future failures. This combined, experimental-numerical method has been used and verified in many studies performed by the authors of this paper [9] and [10].

\section{REFERENCES}

[1] Ismar, H., Burzic, Z., Kapor, N., Kokelj, T. (2012). Experimental investigation of high-strength structural steel welds, Strojniški vestnik - Journal of Mechanical Engineering, vol. 58, no. 6, p. 422-428, DOI: DOI:10.5545/sv-jme.2011.281.

[2] Saoudi, A., Bouazara, M., Marceau, D. (2011). Fatigue failure study of the lower suspension vehicle arm using a multiaxial criterion of the strain energy density. Strojniški vestnik - Journal of Mechanical Engineering, vol. 57, no. 4, p. 345-356, DOI:10.5545/ sv-jme.2009.074.

[3] Arsic, M., Bosnjak, S., Zrnic, N., Sedmak, A., Gnjatovic, N. (2011). Bucket wheel failure caused by residual stresses in welded joints. Engineering Failure Analysis, vol. 18, no. 2, p. 700-712, DOI:10.1016/j. engfailanal.2010.11.009.

[4] Bosnjak, S., Zrnic, N., Simonovic, A., Momcilovic, D. (2009). Failure analysis of the end eye connection of the bucket wheel excavator portal tie-rod support. Engineering Failure Analysis, vol. 16, no. 3, p. 740750, DOI:10.1016/j.engfailanal.2008.06.006.

[5] Rusinski, E, Czmochowski, J, Iluk, A, Kowalczyk, M. (2010). An analysis of the causes of a BWE counterweight boom support fracture. Engineering Failure Analysis, vol. 17, no. 1, p. 179-191, DOI:10.1016/j.engfailanal.2009.06.001.

[6] Rusinski, E., Dragan, S., Moczko, P., Pietrusiak, D. (2012). Implementation of experimental method of determining modal characteristics of surface mining machinery in the modernization of the excavating unit. Archives of Civil and Mechanical Engineering, vol. 12, no. 4, p. 471-476, DOI:10.1016/j.acme.2012.07.002.

[7] Rusiński, E., Czmochowski, J., Smolnicki, T. (2000). Advanced Finite Element Method. Wroclaw University of Technology, Wroclaw. (In Polish)

[8] ISO 5049-1:1994 (1994). Mobile equipment for continuous handling of bulk materials - Part 1: Rules for the design of steel structures. International Organisation for Standardization, Geneva.

[9] Rusiński, E., Moczko, P., Czmochowski, J. (2008). Numerical and experimental analysis of a mine's loader boom crack. Automation in Construction, vol. 17, no. 3, p. 271-277, DOI:10.1016/j.autcon.2007.05.010.

[10] Rusiński, E., Moczko, P. (2010). A combined numerical-experimental method for determining the spatial distribution of a residual stress in a notch. Materials Science-Poland, vol. 28, no. 1, 393-399. 